\title{
RSV bronchiolitis and risk of wheeze and allergic sensitisation in the first year of life
}

\author{
U. Schauer, S. Hoffjan, J. Bittscheidt, A. Köchling, S. Hemmis, S. Bongartz, V. Stephan
}

RSV bronchiolitis and risk of wheeze and allergic sensitisation in the first year of life. U. Schauer, S. Hoffjan, J. Bittscheidt, A.Köchling, S. Hemmis, S. Bongartz, V. Stephan. (C) ERS Journals Ltd 2002.

ABSTRACT: Severe respiratory syncytial virus (RSV) infection has been hypothesised to be a risk factor for the development of allergy and asthma, but epidemiological studies in older children have been inconclusive. The current study hypothesises that the effect of RSV bronchiolitis might be most prominent during the first year after bronchiolitis.

Forty-two infants had experienced RSV bronchiolitis severe enough to cause hospitalisation. For each child with RSV infection, two controls were acquired from a birth cohort and matched for date of birth and sex. All the children were followed prospectively and underwent a follow-up examination at a mean age of $1 \mathrm{yr}$, which included physical examination, and serum immunoglobulin (Ig) $\mathrm{E}$ tests for common food and inhaled allergens.

Risk factors for the development of recurrent wheezing and $\operatorname{IgE}$ antibodies were analysed for the whole group of 126 children. A positive test for IgE antibodies was noted in 14 of $42(33 \%)$ RSV children and in 2 of $84(2.3 \%)$ children in the control group. RSV bronchiolitis was the most important risk factor for allergic sensitisation. Likewise, 13 children $(15.5 \%)$ of the RSV group and three $(3.6 \%)$ children of the control group suffered from recurrent wheezing, and RSV bronchiolitis posed a considerable risk for recurrent wheezing.

Severe respiratory syncytial virus bronchiolitis during the first year of life is an important risk factor for the development of recurrent wheezing and sensitisation to common allergens during the subsequent year.

Eur Respir J 2002; 20: 1277-1283.
Children's Hospital of the Ruhr University, Bochum, Germany.

Correspondence: U. Schauer, Klinik für Kinder- und Jugendmedizin der Ruhr Universität Bochum, St. JosefHospital, Alexandrinenstr. 5, D 44791 Bochum, Germany.

Fax: 492345092612

E-mail: uwe.schauer

@ruhr-uni-bochum.de

Keywords: Allergic sensitisation

bronchiolitis

immunoglobulin $\mathrm{E}$

infant

recurrent wheezing

respiratory syncytial virus infection

Received: March 122002

Accepted after revision: June 302002

This work was supported by grants from the Bundesministerium für Bildung und Forschung (01GC9801).
In infants and toddlers under the age of $2 \mathrm{yrs}$, the virus most frequently isolated during wheezing episodes is respiratory syncytial virus (RSV) [1, 2]. Acute respiratory failure associated with severe bronchospasm, hypoxia, and carbon dioxide retention necessitate hospitalisation of a fraction of $\sim 1-2 \%$ of RSV-infected children [3]. A close link between RSVinduced bronchiolitis and development of asthma has been identified in several studies [4-14]. However, there are conflicting results concerning an association between RSV bronchiolitis and allergic sensitisation. While some studies found bronchiolitis a considerable high-risk factor for allergic sensitisation $[6,8]$, others did not $[11-13,15,16]$. The reason for this apparent contradiction is not clear. Differently recruited control groups, a difference in assessment of atopy and a different severity of RSV-induced illness in the index group [7] were discussed as explanations for conflicting results. In most studies, children were assessed at the age of $8-12$ yrs. It might be possible that different rates of sensitisation at an earlier time point may be missed. Interestingly, there are two studies assessing the same cohort at 1 yr [6] or 5.5 yrs [8] of age for the first time, and at 7 [7] or 10 [9] yrs of age, respectively, for a second time.

Whereas in both studies the first assessment revealed a higher number of allergic sensitisations in the index compared with the control group, at the second assessment the control group caught up with the index group, thereby diminishing the difference in allergic sensitisations. Furthermore, in an early prospective study a viral respiratory infection was seen 1-2 months prior to the onset of sensitisation [17]. Thus, the authors hypothesised that RSV might accelerate the rate of sensitisation and that this effect is seen most clearly in the months after RSV bronchiolitis. The only study, which investigated sensitisation of children with RSV bronchiolitis at $1 \mathrm{yr}$ of age, did not describe a difference between children with and without bronchiolitis at this early time point [6]. However, with respect to food allergens, only egg white was tested. In the current study, a sensitive in vitro multiantigen assay [18] was used which detects, in addition to hen's egg white, sensitisation to five other common food allergens.

\section{Subjects and methods}

\section{Participants}

Ethics. The study was approved by the Research Ethics Committee of the Ruhr University, Bochum, 
Germany. Before enrolment of the participants, informed consent was obtained from the parents.

Infants with respiratory syncytial virus bronchiolitis. From December 1999 to April 2001, 48 children <1 yr of age and without concomitant chronic respiratory, cardiac, or other disease were hospitalised at the Paediatric Dept, St. Josef Hospital, Bochum, Germany, with laboratory-verified RSV bronchiolitis. The clinical diagnosis of acute bronchiolitis required the presence of tachypnoea, wheeze, a prolonged expiratory phase, and crackles on auscultation, recorded at some time during the admission [3]. In addition, many had difficulty with feeding. The diagnosis of RSV infection was made with the ABBOTT testpack RSV (Abbott, Wiesbaden, Germany), an enzyme immunoassay for the rapid detection of RSV from nasopharyngeal aspirates. The parents were asked to participate with their infants in a follow-up study of the development of respiratory and atopic diseases. The parents of 42 infants (18 males) agreed to participate in the study. None of them had respiratory distress during the neonatal period. One child had symptoms of atopic dermatitis (AD) before the RSV infection. Three infants had experienced an episode of wheezing 4 weeks before the RSV bronchiolitis. The mean age of the children when admitted to hospital for RSV bronchiolitis was 16 weeks (range 5-42 weeks). Thirtyfive $(83 \%)$ of the children were given bronchodilators by inhalation. Antibiotic therapy was administered to 21 children $(50 \%)$. Twenty-three children $(55 \%)$ needed oxygen for 4 days (range $1-10$ days) and $3(7 \%)$ had to be ventilated for 3 days (range 1-6 days). All children were examined during the infection by one of the authors. Their mean hospital stay was 10 days (range 3-26 days).

Control group. Children were selected from a cohort of children followed from birth. Families lived in the catchment area of the hospital. For each child with RSV bronchiolitis, two children with birth dates closest to the index children, and of the same sex were included. The control group thus consisted of 84 infants (36 males). The mean difference in the birth dates between the RSV children and their matched controls was 34 days (range 6-92 days). No complications during the neonatal period were reported. None of the controls had been hospitalised for respiratory symptoms between the neonatal period and their first examination.

Exclusion criteria. Children with neonatal respiratory distress and children who were born before the 38th week of gestation were excluded from the study.

\section{Study design}

The children from the RSV group were re-examined after 6-9 months at a mean age of $1 \mathrm{yr}$ (range $0.7-1.5 \mathrm{yrs}$ ). The children from the control group were examined at the same times, resulting in a mean age of $1 \mathrm{yr}$ (range 0.7-1.6 yrs). All children were examined by one of the authors and recordings of height and weight were taken. The parents were interviewed using a structured questionnaire. Heredity for atopic disease in parents and siblings, smoking habits of the parents (current and during pregnancy), presence of indoor furred animals, and duration of breastfeeding were recorded. At follow-up, serum immunoglobulin (Ig) G antibodies against RSV were analysed using enzyme-linked immunosorbent assay (Genzyme Virotech, Rüsselsheim, Germany). Values of $>6$ Units were regarded as positive. In addition, serum $\operatorname{IgE}$ antibodies to foods and inhalants were determined using two screening tests for IgE antibodies (Fx5 and Sx1, CAP system; Pharmacia, Freiburg, Germany) according to the instructions given by the manufacturer [18]. The Fx5 test detects antibodies to egg white, soya protein, cow's milk, wheat, peanut, and fish allergens and the Sxl test identifies antibodies against timothy, rye, birch, mugwort, house-dust mite, cat, dog and moulds. Values of $\geqslant 0.35 \mathrm{kU} \cdot \mathrm{L}^{-1}$ which have been shown previously to be of diagnostic value [19], were regarded as positive.

AD was defined as a pruritic, chronic, or chronically relapsing noninfectious dermatitis, based on the suggestions of HANIFIN [20]. The term "recurrent wheezing" was used in case of three episodes of bronchial obstruction. Atopic disease in parents or siblings signifies that they had been diagnosed at some time by a physician with any of the following: asthma, allergic rhinoconjunctivitis, or AD. Single heredity for asthma or atopic disease signifies disease in one parent or sibling. Double heredity indicates disease in two or more parents and/or siblings.

\section{Statistics}

For comparisons between groups Fisher's exact test was used for discrete variables and the Mann-Whitney U-test for continuous variables. A value of $\mathrm{p}<0.05$ was regarded as significant. To determine which hereditary and environmental risk factors were important for the development of bronchial obstructive symptoms and sensitisation in the whole group of 126 children, Fisher's exact test between each risk factor and each variable was first performed. Odds ratios (OR) and 95\% confidence intervals (CI) were also calculated. To estimate which risk factors were independently related to the bronchial symptoms and sensitisation, the risk factors with $\mathrm{p}<0.10$ in this test were included in multivariate forward stepwise logistic regression analyses. ORs and 95\% CI were also calculated for significant risk factors in this model.

\section{Results}

Respiratory syncytial virus bronchiolitis is a risk factor for recurrent wheezing

In this study, 42 children hospitalised for RSV bronchiolitis were followed prospectively and compared to 84 children without bronchiolitis. Serum IgG antibodies to RSV were found in 39 of $42(93 \%) \mathrm{RSV}$ 
and in 29 of $84(35 \%)$ control children $(\mathrm{p}<0.0001$; OR $24.66 ; 95 \%$ CI 7.01-86.73). All of the 29 controls with $\mathrm{IgG}$ antibodies had a history of a mild respiratory infection (in eight cases combined with wheezing) which did not require hospitalisation at the time of the RSV epidemic. The frequency of AD was comparable in the RSV children and the controls.

The RSV children and the control group were similar for 11 of 15 background factors, including the family history of atopy/asthma. There were significant differences in the four diverging factors, smoking by the father, by the mother, and during pregnancy, as well as number of siblings (table 1). The frequency of recurrent wheezing was significantly higher for the RSV children at follow-up (table 2). Five of the RSV children and none of the controls had both recurrent wheezing and positive tests. Several possible hereditary and environmental risk factors for the development of different bronchial obstructive symptoms were evaluated using Fisher's exact test (table 3). RSV bronchiolitis, current smoking by the mother and during pregnancy were the most important risk factors for recurrent wheezing. The risk factors in table 3 with a p-value $<0.10$ were entered into a forward stepwise logistic regression analysis to estimate which factors were independently related to the development of obstructive symptoms and sensitisation (table 4). RSV bronchiolitis and smoking in pregnancy were independent risk factors for recurrent wheezing. In addition, smoking by the mother and male sex were risk factors for wheezing.

\section{Respiratory syncytial virus bronchiolitis is a risk} factor for atopic sensitisation

At the follow-up a positive test to allergen was seen in 14 of 42 RSV children and in two of 84 control children. Thirteen children were allergic to food antigens and one to inhalant allergens alone. Two children from the RSV group were allergic to both food and inhalant allergens.

In addition to RSV bronchiolitis, current smoking by the mother and smoking during pregnancy, as well as double heredity of atopy and male sex were single risk factors for the development of sensitisation (table 3). To estimate which risk factors were independently related to allergic sensitisation a forward stepwise logistic regression analysis was

Table 1. - Evaluation of background factors in 42 children with respiratory syncytial virus (RSV) bronchiolitis and 84 control children at $1 \mathrm{yr}$ of age

\begin{tabular}{lccc}
\hline Background Factor & RSV group & Control group & p-value \\
\hline Single heredity for atopy & 17 & 49 & 0.09 \\
Double heredity for atopy & 6 & 9 & 0.57 \\
Heredity for asthma & 9 & 13 & 0.46 \\
Double heredity for asthma & 4 & 5 & 0.48 \\
Male sex & 18 & 36 & 1.00 \\
Smoking & 18 & 20 & 0.04 \\
By father & 18 & 13 & $<0.01$ \\
By mother & 16 & 9 & 0.001 \\
During pregnancy & 9 & 20 & $<.83$ \\
Indoor furred animals & $1.33 \pm 1.16$ & $0.51 \pm 0.75$ & 0.0001 \\
Number of siblings & $39.3 \pm 1.3$ & $39.6 \pm 1.1$ & 0.60 \\
Mean duration of pregnancy & $3456 \pm 368$ & $3510 \pm 399$ & 0.98 \\
Mean birth weight g & $1.9 \pm 2.6$ & $2.0 \pm 2.8$ & 0.85 \\
Breastfeeding month & $9.6 \pm 0.9$ & $9.7 \pm 1.1$ & 0.56 \\
Mean weight at follow-up kg & $76.0 \pm 3.0$ & $75.6 \pm 3.2$ & \\
Mean height at follow-up cm & & & \\
\hline
\end{tabular}

Data are presented as $\mathrm{n}$ or mean $\pm \mathrm{SD}$.

Table 2. - Number of children with bronchial obstructive symptoms, atopic dermatitis and allergic sensitisation at $1 \mathrm{yr}$ of age in the respiratory syncytial virus (RSV) bronchiolitis and control groups

\begin{tabular}{|c|c|c|c|c|c|}
\hline \multirow[t]{2}{*}{ Symptom } & \multirow[t]{2}{*}{ RSV group } & \multirow[t]{2}{*}{ Control group } & \multicolumn{3}{|c|}{ Significance } \\
\hline & & & p-value & OR & $95 \%$ CI \\
\hline Total subjects & 42 & 84 & & & \\
\hline Wheezing & 28 & 13 & $<0.001$ & 10.92 & $4.56-26.14$ \\
\hline Recurrent wheezing $\#$ & 13 & 3 & $<0.001$ & 12.10 & $3.22-45.55$ \\
\hline Atopic dermatitis & 7 & 16 & 0.81 & 0.85 & $0.32-2.26$ \\
\hline $\operatorname{IgE}>0.35 \mathrm{kU} \cdot \mathrm{L}^{-1}$ & 14 & 2 & $<0.001$ & 20.50 & $4.38-95.89$ \\
\hline Food-specific IgE & 14 & 1 & $<0.001$ & 56.44 & $7.15-445.59$ \\
\hline Inhalant-specific IgE & 2 & 1 & 0.26 & 6.38 & $0.64-63.39$ \\
\hline
\end{tabular}

Data are presented as $\mathrm{n}$ unless otherwise stated. OR: odds ratio; CI: confidence interval; Ig: immunoglobulin; U: units. \#: three episodes of bronchial obstruction. 
Table 3.-Univariate test of various possible risk factors for wheezing and allergic sensitisation at $1 \mathrm{yr}$ of age using Fisher's exact test in 42 children with respiratory syncytial virus (RSV) bronchiolitis and 84 controls

\begin{tabular}{|c|c|c|c|c|c|c|c|c|c|}
\hline \multirow[t]{2}{*}{ Risk factor } & \multicolumn{3}{|c|}{ Wheezing } & \multicolumn{3}{|c|}{ Recurrent wheezing } & \multicolumn{3}{|c|}{ Sensitisation } \\
\hline & p-value & OR & $95 \% \mathrm{CI}$ & p-value & OR & $95 \% \mathrm{CI}$ & p-value & OR & $95 \% \mathrm{CI}$ \\
\hline RSV bronchiolitis & $<0.0001$ & 10.92 & $4.56-26.14$ & $<0.0001$ & 12.10 & $3.22-45.55$ & $<0.0001$ & 20.50 & $4.38-95.89$ \\
\hline Mild RSV infection & 0.65 & 0.74 & $0.30-1.85$ & 0.36 & 0.44 & $0.09-2.06$ & 0.12 & 0.20 & $0.02-1.55$ \\
\hline \multicolumn{10}{|l|}{ Smoking } \\
\hline By father & 0.02 & 2.03 & $1.15-5.63$ & 0.25 & 1.98 & $0.68-5.79$ & 0.5626 & 1.46 & $0.49-4.36$ \\
\hline By mother & $<0.0001$ & 6.41 & $2.65-15.46$ & $<0.01$ & 5.14 & $1.72-15.34$ & $<0.0001$ & 9.90 & $3.09-31.68$ \\
\hline During pregnancy & $<0.01$ & 4.33 & $1.73-10.82$ & $<0.0001$ & 10.56 & $3.34-33.32$ & $<0.0001$ & 10.56 & $3.34-33.32$ \\
\hline Furred animals & 0.82 & 1.12 & $0.47-2.69$ & 0.76 & 1.13 & $0.34-3.83$ & 0.12 & 0.20 & $0.02-1.55$ \\
\hline Heredity asthma & 0.21 & 0.77 & $0.77-5.02$ & 0.48 & 1.70 & $0.49-5.88$ & 1.00 & 1.11 & $0.29-4.26$ \\
\hline Double heredity asthma & 0.15 & 2.81 & $0.71-11.10$ & 0.32 & 2.10 & $0.40-11.14$ & 1.00 & 0.42 & $0.05-3.44$ \\
\hline Heredity atopy & 1.00 & 0.93 & $0.44-1.97$ & 0.79 & 1.20 & $0.42-3.44$ & 0.28 & 0.98 & $0.20-4.78$ \\
\hline Double heredity atopy & 0.15 & 0.73 & $0.22-2.44$ & 0.41 & 1.88 & $0.47-7.58$ & $<0.01$ & 6.00 & $1.80-19.98$ \\
\hline Male sex & 0.06 & 2.22 & 1.044 .47 & 0.79 & 0.77 & $0.26-2.27$ & $<0.01$ & 4.86 & $1.47-16.05$ \\
\hline More than one sibling & $<0.01$ & 4.47 & $1.23-16.23$ & 0.03 & 3.80 & $1.20-12.00$ & 0.14 & 1.67 & $0.82-8.72$ \\
\hline
\end{tabular}

OR: odds ratio; CI: confidence interval.

Table 4. - Multivariate test of possible risk factors for wheezing and allergic sensitisation at $1 \mathrm{yr}$ of age using logistic regression in 42 children with respiratory syncytial virus (RSV) bronchiolitis and 84 controls\#

\begin{tabular}{|c|c|c|c|c|c|c|c|c|c|}
\hline \multirow[t]{2}{*}{ Risk factor } & \multicolumn{3}{|c|}{ Wheezing } & \multicolumn{3}{|c|}{ Recurrent wheezing } & \multicolumn{3}{|c|}{ Sensitisation } \\
\hline & p-value & OR & $95 \% \mathrm{CI}$ & p-value & OR & $95 \% \mathrm{CI}$ & p-value & OR & $95 \% \mathrm{CI}$ \\
\hline RSV bronchiolitis & $<0.0001$ & 10.32 & $3.56-29.96$ & 0.01 & 8.92 & $1.42-55.88$ & $<0.001$ & 20.66 & $3.53-120.75$ \\
\hline Smoking & & & & & & & & & \\
\hline By father & 0.85 & 1.12 & $0.36-3.46$ & & & & & & \\
\hline By mother & 0.01 & 8.42 & $1.51-46.81$ & 0.68 & 0.68 & $0.11-4.12$ & 0.04 & 9.17 & $1.17-72.21$ \\
\hline During pregnancy & 0.41 & 0.50 & $0.10-2.60$ & 0.02 & 6.45 & $1.52-27.23$ & 0.79 & 1.31 & $0.19-9.10$ \\
\hline Double heredity atopy & & & & & & & 0.59 & 1.31 & $0.25-11.58$ \\
\hline Male sex & $<0.01$ & 4.24 & $1.49-12.05$ & & & & $<0.01$ & 12.2 & $2.37-62.5$ \\
\hline More than one sibling & 0.19 & 2.25 & $0.68-7.46$ & 0.29 & 2.14 & $0.52-8.81$ & & & \\
\hline
\end{tabular}

OR: odds ratio; CI: confidence interval. " : risk factors with a p-value $<0.10$ in Fisher's exact test (from table 3 ) were entered.

performed (table 4). RSV bronchiolitis was the most important independent risk factor for sensitisation followed by male sex and current smoking by the mother. The number of siblings turned out to have no influence on sensitisation in this study.

\section{Discussion}

In the present study, children hospitalised with RSV bronchiolitis displayed a significantly higher rate of recurrent wheezing and allergic sensitisation only a few months after the RSV infection. Forty-two children with RSV bronchiolitis were followed during the first month of life and compared to 84 control children. The index children suffered from severe RSV infection necessitating hospitalisation. This group of children usually only comprises $\sim 1 \%$ of all children infected by RSV [21]. Like 35\% of control children with positive anti-RSV IgG, most of the children suffered from a benign upper respiratory tract infection after contact with RSV. The two study groups differed in number of siblings and smoking in the family. Both factors may predispose for RSV bronchiolitis. An infant's older sibling is most likely to introduce the virus into the family [22], and the number of persons sharing the room has been identified as a risk factor for RSV bronchiolitis [23]. In the same way, the risk for RSV infections is increased in children attending daycare centres [23]. Since daycare is not institutionalised in western Germany, this point was not evaluated in this study. Smoking [6, 8, 9], in particular prenatal smoking by the mother [24], is a known predisposing factor for RSV bronchiolitis. Maternal smoking during pregnancy is an important determinant of lung function shortly after birth, and this may explain the strong link between smoke exposure in utero and subsequent risk of lower respiratory tract illness [25].

The main question of the study however was whether RSV bronchiolitis poses a risk for subsequent wheezing and allergic sensitisation. Multivariate analysis in the whole group of 126 children showed that RSV bronchiolitis was the single most important risk factor for wheezing and recurrent wheezing. This was followed by smoking during pregnancy for wheezing and recurrent wheezing, and by male sex for wheezing. In this regard, the present study 
confirms the result of many others [4-13]. Likewise, smoking in the family $[6,8,9]$, and in particular smoking during pregnancy [24], were previously identified as risk factors for wheezing. In addition, male sex was identified as a risk factor for wheezing. Smoking and male sex were found to predispose for decreased maximum expiratory flow in the first year of life [24]. In a prospective study, lower levels of lung function were observed before the development of any lower respiratory illness in children with recurrent wheezing [26]. Thus, severe RSV bronchiolitis and recurrent wheezing may be confined to children with narrow airways and a higher risk of wheeze. On the other hand, it has been shown that an increased incidence of wheezing after RSV infection is not only restricted to children with severe RSV bronchiolitis, but also to children with mild disease not requiring hospitalisation $[15,16]$. Therefore, it cannot be ruled out that RSV infection actively induces bronchial hyperreactivity. One possible mechanism for induction of hyperreactivity might be induction of allergies.

In the present study, children hospitalised with RSV bronchiolitis displayed a significantly higher rate of allergic sensitisation only a few months after RSV bronchiolitis. RSV bronchiolitis was the single most important risk factor for sensitisation. Additional risk factors were male sex and smoking by the mother. These risk factors have been identified previously in some [27], but not all [28] studies. It is possible that these additional risk factors might predispose for RSV-mediated immunmodulation and therefore were not identified in other studies focusing on risk factors for allergy in general [29]. Sensitisations found in this study were mainly directed to food antigens. The only study which investigated sensitisation of children with RSV bronchiolitis at $1 \mathrm{yr}$ of age, did not describe a difference between children with and without bronchiolitis at this early time point [6]. With respect to food allergens, only egg white was tested. In the current study, a sensitive in vitro multiantigen assay [18] was used which detects, in addition to hen's egg white, sensitisation to five other common food allergens. Even though the diagnostic value might be higher if sensitisation to food was detected by single allergen specific tests or skin-prick tests against common allergens [19], the results of this study shed some light on the mechanisms of RSV-induced allergic sensitisation. The predominance of food sensitisation in infants after RSV bronchiolitis is consistent with the notion that RSV bronchiolitis may accelerate the rate of sensitisation. The IgE responses during the first months of life are commonly directed to food proteins [29]. Children with food allergies in infancy are prone to sensitisation to environmental allergens between the first and tenth birthdays [30]. Thus, the increased rate of sensitisation to food antigen found in this study at $1 \mathrm{yr}$ of age may predispose to a higher rate of sensitisation against inhalant allergens at later time points, as described in other studies of children with RSV bronchiolitis [6-8].

The concept that RSV bronchiolitis accelerates allergic sensitisation is further supported by the fact that the relative risk for sensitisation was as high as 20.66 in this study at $1 \mathrm{yr}$ of age, whereas in children examined by SIGURS and co-workers [6, 7], it was 3.6 at $3 \mathrm{yrs}$ of age and 2.4 at $7.5 \mathrm{yrs}$. In these studies, the declining influence of RSV bronchiolitis was due to an increase in allergic sensitisations in the control group. In extrapolating the present data to later time points, it might be speculated that the sensitisation rate of the control group will further increase to a level of $25 \%$, as seen in the general population of developed countries [31], and thus level off with the RSV group. If this is true then it is not surprising that a number of retrospective studies with older children were unable to detect any influence of RSV on allergic sensitisation $[4,5,11-13]$.

The high rate of sensitisation to food, but not to inhalant allergens, in this study might also shed some light on the pathophysiology of RSV-associated increase in allergic sensitisation. The reaction to food antigens is likely to be either systemic or to be confined to the gut [32] rather than to the respiratory system. In accordance with other studies [6, 7, 16], the authors found that only severe RSV bronchiolitis but not a mild RSV infection increased the risk of sensitisation. In severe bronchiolitis, RSV was shown to spread outside the respiratory tract [33]. In addition, a modulation of the systemic immune response was demonstrated by several authors [34-36]. It is thus conceivable that a severe RSV infection might be able to modulate the immune response in organ systems other than the respiratory tract. Furthermore, virusinduced immune modulation is most prominent during the acute infection and the weeks thereafter, as shown in murine RSV infection [37, 38] or human measles infection [39]. It is therefore likely, that its effect on allergic sensitisation is most clearly seen shortly after the infection.

The predominance of sensitisation to food rather than to inhalant allergens makes a direct relation between sensitisation and respiratory obstructive symptoms very unlikely. This corresponds with the observation reported by STEIN et al. [16] that an increase of bronchial obstructive disease is found in children with mild RSV infection not requiring hospitalisation and that this increased rate of obstructive disease is not related to allergic sensitisation.

Although the current study may help to clarify the association between respiratory syncytial virus infection, recurrent wheezing and allergic sensitisation, the limitations of the study need to be made clear. The study groups were not matched according to smoking in the family and number of siblings. Only children with severe bronchiolitis but not children with milder lower respiratory tract infections were compared to healthy controls. Instead of single allergen tests, a more insensitive multiallergen test was used, and the children were only followed during the first year. These problems have to be addressed in future studies.

\section{References}

1. Johnston SL. The role of viral and atypical bacterial pathogens in asthma pathogenesis. Pediatr Pulmonol Suppl 1999; 18: 141-143.

2. McIntosh K, Ellis EF, Hoffman LS, Lybass TG, 
Eller JJ, Fulginiti VA. The association of viral and bacterial respiratory infections with exacerbations of wheezing in young asthmatic children. J Pediatr 1973; 82: 578-590.

3. Simoes EA. Respiratory syncytial virus infection. Lancet 1999; 354: 847-852.

4. Mok JY, Simpson H. Outcome of acute lower respiratory tract infection in infants: preliminary report of seven-year follow-up study. BMJ 1982; 285: 333-337.

5. Mok JY, Simpson H. Outcome for acute bronchitis, bronchiolitis, and pneumonia in infancy. Arch Dis Child 1984; 59: 306-309.

6. Sigurs N, Bjarnason R, Sigurbergsson F, Kjellman B, Bjorksten B. Asthma and immunoglobulin E antibodies after respiratory syncytial virus bronchiolitis: a prospective cohort study with matched controls. Pediatrics 1995; 95: 500-505.

7. Sigurs N, Bjarnason R, Sigurbergsson F, Kjellman B. Respiratory syncytial virus bronchiolitis in infancy is an important risk factor for asthma and allergy at age 7. Am J Respir Crit Care Med 2000; 161: 1501-1507.

8. Murray M, Webb MS, O'Callaghan C, Swarbrick AS, Milner AD. Respiratory status and allergy after bronchiolitis. Arch Dis Child 1992; 67: 482-487.

9. Noble V, Murray M, Webb MS, Alexander J, Swarbrick AS, Milner AD. Respiratory status and allergy nine to 10 years after acute bronchiolitis. Arch Dis Child 1997; 76: 315-319.

10. Osundwa VM, Dawod ST, Ehlayel M. Recurrent wheezing in children with respiratory syncytial virus (RSV) bronchiolitis in Qatar. Eur J Pediatr 1993; 152: 1001-1003.

11. Pullan CR, Hey EN. Wheezing, asthma, and pulmonary dysfunction 10 years after infection with respiratory syncytial virus in infancy. BMJ 1982; 284 : $1665-$ 1669.

12. Sims DG, Gardner PS, Weightman D, Turner MW, Soothill JF. Atopy does not predispose to RSV bronchiolitis or postbronchiolitic wheezing. $B M J$ 1981; 282: 2086-2088.

13. Sims DG, Downham MA, Gardner PS, Webb JK, Weightman D. Study of 8-year-old children with a history of respiratory syncytial virus bronchiolitis in infancy. BMJ 1978; 1: 11-14.

14. Wennergren G, Kristjansson S. Relationship between respiratory syncytial virus bronchiolitis and future obstructive airway diseases. Eur Respir J 2001; 18: 1044-1058.

15. McConnochie KM, Roghmann KJ. Bronchiolitis as a possible cause of wheezing in childhood: new evidence. Pediatrics 1984; 74: 1-10.

16. Stein RT, Sherrill D, Morgan WJ, et al. Respiratory syncytial virus in early life and risk of wheeze and allergy by age 13 years. Lancet 1999; 354: 541-545.

17. Frick OL, German DF, Mills J. Development of allergy in children. I. Association with virus infections. J Allergy Clin Immunol 1979; 63: 228-241.

18. Wood RA, Schuberth KC, Sampson HA. Value of a multiantigen radioallergosorbent test in diagnosing atopic disease in young children. J Pediatr 1990; 117: 882-885.

19. Kulig M, Bergmann R, Niggemann B, Burow G, Wahn U. Prediction of sensitization to inhalant allergens in childhood: evaluating family history, atopic dermatitis and sensitization to food allergens.
The MAS Study Group. Multicentre Allergy Study. Clin Exp Allergy 1998; 28: 1397-1403.

20. Hanifin JM. Basic and clinical aspects of atopic dermatitis. Ann Allergy 1984; 52: 386-395.

21. Sigurs N. Epidemiologic and clinical evidence of a respiratory syncytial virus - reactive airway disease link. Am J Respir Crit Care Med 2001; 163: S2-S6.

22. Hall CB, Geiman JM, Biggar R, Kotok DI, Hogan PM, Douglas GR Jr. Respiratory syncytial virus infections within families. $N$ Engl J Med 1976; 294: 414 419.

23. Holberg CJ, Wright AL, Martinez FD, Ray CG, Taussig LM, Lebowitz MD. Risk factors for respiratory syncytial virus-associated lower respiratory illnesses in the first year of life. Am $J$ Epidemiol 1991; 133: 1135-1151.

24. Young S, Sherrill DL, Arnott J, Diepeveen D, LeSouef PN, Landau LI. Parental factors affecting respiratory function during the first year of life. Pediatr Pulmonol 2000; 29: 331-340.

25. Tager IB, Hanrahan JP, Tosteson TD, et al. Lung function, pre- and post-natal smoke exposure, and wheezing in the first year of life. Am Rev Respir Dis 1993; 147: 811-817.

26. Young S, O'Keeffe PT, Arnott J, Landau LI. Lung function, airway responsiveness, and respiratory symptoms before and after bronchiolitis. Arch Dis Child 1995; 72: 16-24.

27. Burr ML, Merrett TG, Dunstan FD, Maguire MJ. The development of allergy in high-risk children. Clin Exp Allergy 1997; 27: 1247-1253.

28. Strachan DP, Cook DG. Health effects of passive smoking. 5. Parental smoking and allergic sensitisation in children. Thorax 1998; 53: 117-123.

29. Wahn U. What drives the allergic march? Allergy 2000; 55: 591-599.

30. Nickel R, Kulig M, Forster J, et al. Sensitization to hen's egg at the age of twelve months is predictive for allergic sensitization to common indoor and outdoor allergens at the age of three years. J Allergy Clin Immunol 1997; 99: 613-617.

31. International Study of Asthma and Allergies in Childhood. Worldwide variation in prevalence of symptoms of asthma, allergic rhinoconjunctivitis, and atopic eczema: ISAAC. The International Study of Asthma and Allergies in Childhood (ISAAC) Steering Committee. Lancet 1998; 351: 1225-1232.

32. Eigenmann P, Tropia L, Hauser C. The mucosal adhesion receptor [alpha]4[beta]7 integrin is selectively increased in lymphocytes stimulated with [beta]lactoglobulin in children allergic to cow's milk. J Allergy Clin Immunol 1999; 103: 931-936.

33. O'Donnell DR, McGarvey MJ, Tully JM, Balfour-Lynn IM, Openshaw PJ. Respiratory syncytial virus RNA in cells from the peripheral blood during acute infection. J Pediatr 1998; 133: 272-274.

34. Bendelja K, Gagro A, Bace A, et al. Predominant type-2 response in infants with respiratory syncytial virus (RSV) infection demonstrated by cytokine flow cytometry. Clin Exp Immunol 2000; 121: 332-338.

35. Roman M, Calhoun WJ, Hinton KL, et al. Respiratory syncytial virus infection in infants is associated with predominant Th-2-like response. Am J Respir Crit Care Med 1997; 156: 190-195.

36. Aberle JH, Aberle SW, Dworzak MN, et al. Reduced interferon-gamma expression in peripheral blood mononuclear cells of infants with severe respiratory 
syncytial virus disease. Am J Respir Crit Care Med 1999; 160: 1263-1268.

37. Schwarze J, Hamelmann E, Bradley KL, Takeda K, Gelfand EW. Respiratory syncytial virus infection results in airway hyperresponsiveness and enhanced airway sensitization to allergen. J Clin Invest 1997; 100: 226-233.
38. O'Donnell DR, Openshaw PJ. Anaphylactic sensitization to aeroantigen during respiratory virus infection. Clin Exp Allergy 1998; 28: 1501-1508.

39. Tamashiro VG, Perez HH, Griffin DE. Prospective study of the magnitude and duration of changes in tuberculin reactivity during uncomplicated and complicated measles. Pediatr Infect Dis $J$ 1987; 6: 451-454. 